\title{
DIFFRACTION OF THE GAUSSIAN BEAM ON LAYERED LENS AND SIMILAR A CONICAL AND DIFFRACTION AXICONS
}

\author{
D.A. Savelyev ${ }^{1,2}$ \\ ${ }^{1}$ Samara National Research University, Samara, Russia \\ ${ }^{2}$ Image Processing Systems Institute - Branch of the Federal Scientific Research Centre "Crys- \\ tallography and Photonics" of Russian Academy of Sciences, Samara, Russia
}

\begin{abstract}
In this paper we consider the possibility of replacing the diffraction axicon and the conical axicon on the gradient lens with a linear variation of the refractive index. Analytically and numerically using the finite-difference timedomain method we performed a comparative study of the Gaussian beam diffraction on diffraction mikro-axicon, conical axicon and gradient microlens consisting of subwavelength layers. The parameters under consideration the types of elements estimated in the depth of focus and a transverse dimension of beam.
\end{abstract}

Keywords: diffraction optics, subwavelength structures, laser beams, diffraction axicon, layered lens, conical axicon, FDTD.

Citation: Savelyev DA. Diffraction of the Gaussian beam on layered lens and similar a conical and diffraction axicons. CEUR Workshop Proceedings 2016; 1638: 117-124. DOI: 10.18287/1613-0073-2016-1638-117-124

\section{Introduction}

Environments with light propagates in curved paths are the subject of gradient optics (GRIN - GRadient INdex) [1]. The flat surfaces of gradient lenses make them very useful for collimating light from the end of single mode fiber and focusing of the collimated beam to another single mode fiber [2]. Thus, light beams passing through the gradient lens can be the use for better focusing [3-5].

When transmitting information over optical fibers easier connection between the fibers do using gradient elements [6,7], usually, such components are in some way analogue of a lens $[8,9]$, which forms a short focus. Typically used two gradient elements with a sufficiently precise mutual agreement: one at the output, which scatters the laser beams and one at the entrance, which collects the laser beam [10,11].

One advantage of using the axicon is the formation of an extended focus [12, 13], including subwavelength lateral size $[14,15]$. The advantage of using a diffrac-tion axicon before the conical axicon is in the relative simplicity of manufacturing, and in the possibility of achieving, for this element of high numerical aperture values, inac- 
cessible to the conical axicon due to total internal reflection [16-18]. An extended focus [19] can be used to alleviate the requirements for alignment of the optical fiber connection.

For connections required flat edge [20], and diffraction axicon has it. In this pa-per, we consider particularly focusing Gaussian beams by using gradient optical ele-ments $[21,22]$ and similar a conical and diffraction axicons. For the numerical simu-lation of diffraction considered laser beams used finite-difference time-domain method (FDTD) using high-performance computing [23].

\section{Diffraction of the Gaussian beam}

Under the linear change of the refractive index $n(r)=n_{0}(1-\alpha r)$ the phase difference is analogous to a conical axicon:

$$
\Delta \varphi_{\text {lin }}(r)=-k n_{0} L \alpha r
$$

where $n_{0}$ - the refractive index in the center, $k=2 \pi / \lambda, \lambda$ is the wavelength, $L-$ lens thickness, $\alpha$ - parameter governing the rate of change of the refractive index. Let's define parameters of a conical axicon creating the same phase difference [14]:

$$
\Delta \varphi_{a x}(r)=-k\left(n_{a x}-1\right) \cdot r \cdot \operatorname{ctg} \beta,
$$

In according to equations (1) and (2), we selected axicon angle:

$\beta=\operatorname{arctg}\left(\frac{n_{a x}-1}{\alpha L n_{0}}\right)$,

where nax - the refractive index of axicon material, $\beta$ - a half of angle at the axicon tip (Fig1(c)). $\mathrm{H}$ - axicon height:

$$
H=\frac{R}{\operatorname{tg} \beta},
$$

Let us consider the diffraction axicon (Fig.1 (b)). The phase difference between the central ray and a ray extending from the center at a distance is equal:

$$
\Delta \varphi_{\text {диф }}=-\mathrm{k} \cdot \mathrm{NA} \cdot \mathrm{r}
$$

where NA - the numerical aperture of the axicon, $r$ - radius of the axicon. Then, the numerical aperture of axicon is

$$
\mathrm{NA}=\mathrm{n}_{0} \mathrm{~L} \alpha
$$

where $n 0=3,47$ - the value of the central layer for the considered layered lens. Period of axicon $\mathrm{d}$ is changed to the following law: 


$$
\mathrm{d}=\frac{\lambda}{\mathrm{NA}},
$$

Height axicon considered on the basis of the phase shift on $\pi$ :

$$
\mathrm{h}=\frac{\pi}{\mathrm{k}\left(\mathrm{n}_{\mathrm{ax}}-1\right)}=\frac{\lambda}{2\left(\mathrm{n}_{\mathrm{ax}}-1\right)} \approx 0,21 \lambda,
$$

when the refractive index $n_{a x}=3,47$.

a)

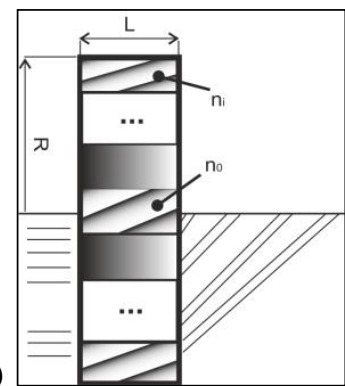

b)

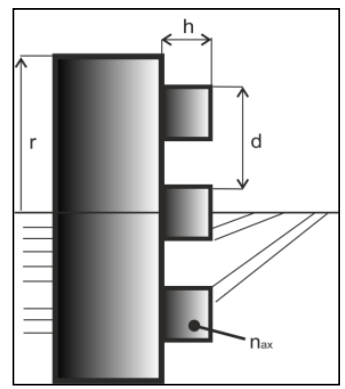

c)

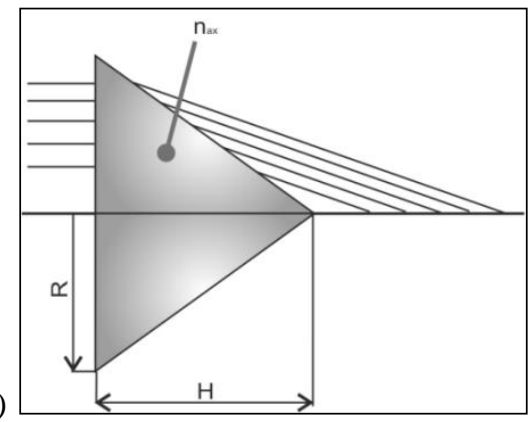

Fig. 1. The transverse structure (scheme) of the matched linearly layered lens (a), diffractive axicon (b) and the conical axicon (c)

Simulation parameters: the wavelength $\lambda=1.55$ microns, the size of the computational domain $\mathrm{x}, \mathrm{y}, \mathrm{z} \in[-4,5 \lambda ; 4,5 \lambda]$. The thickness of the absorbing layer PML $\sim 0.65 \lambda$ ( 1 micron), the sampling step of space $-\lambda / 31$, the sampling step of time $-\lambda /(62 c)$, where $\mathrm{c}$ is the velocity of light. As the input laser radiation with the circular polarization we use the fundamental Gaussian mode. In the case of layered lens we use the linearly changes of refractive index of lens: from $n=3.47$ in center to $n=1.34$ at the lens edge. Let's denote a lens width on propagation axis of the laser beam as L.

Numerical simulation was made using the computational cluster with the power of 775 GFlops. The cluster's characteristics are the following: 116 cores, computing nodes -7 dual servers HP ProLiant 2xBL220c, RAM volume 112 Gbit. 
We consider the half width at half intensity (FWHM) and depth of field (DOF). Fix a lens width $\mathrm{L}=1,55 \lambda$ with refractive index $\mathrm{n}=3,47$. The numerical results studies for the axicon and the layered lenses with a corresponding $\alpha$ are given in Table 1 .

Table 1. Diffraction of Gaussian beam on a layered lens, diffraction and conical axicon

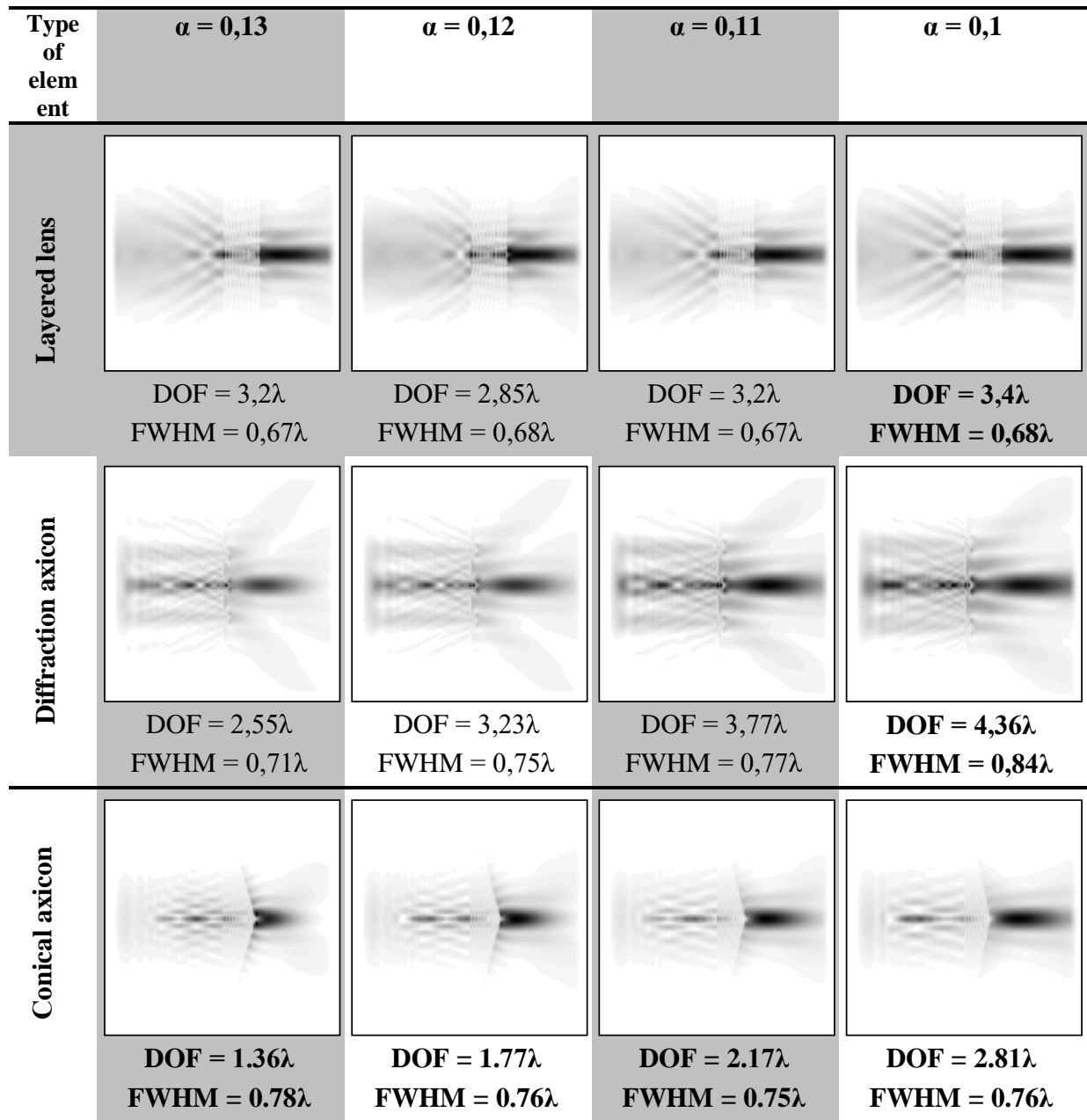

Reducing the parameter $\alpha$ for a layered lens increases the length of the light segment with a substantially constant radius of the light spot. A separate case with $\alpha=0.12$, where the observed change in the overall diffraction patterns and reducing the depth of focus.

For diffraction axicon situation is as follows: reduction in $\alpha$ (that means reducing the numerical aperture) also leads to an increase in the length of the light segment. No cases like the case $\alpha=0.12$ for a layered lens. And also we see expected focal spot size increases.

For the conical axicon also decrease $\alpha$ (which is equivalent to an increase of the angle $\beta$ ) leads to elongation of the light segment. But in this case also seems certain number 
$\alpha=0.11$, where the focal spot is minimal. Reduction of $\alpha$ leads to a broadening of the beam.

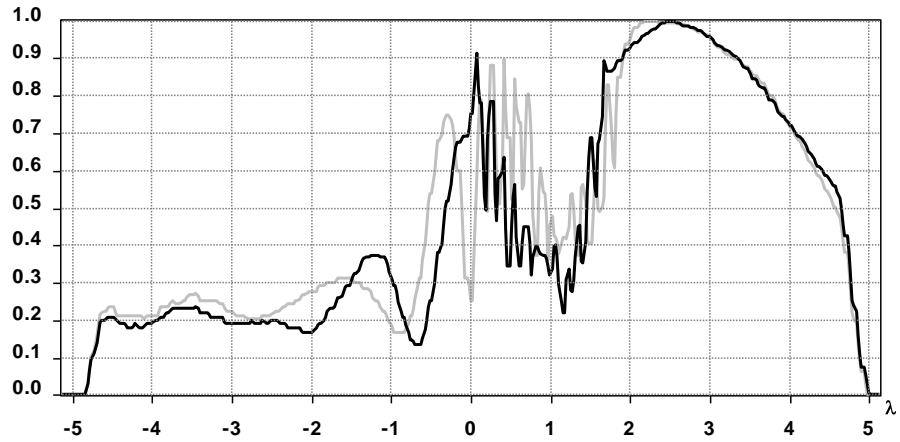

Fig. 2. Diffraction of Gaussian beam on a layered lens with changing $L(\alpha=0.11)$, the intensity: $\mathrm{L}=1.55 \lambda$ (black line), $\mathrm{L}=1.75 \lambda$ (gray line)

Table 2. Results of numerical simulation when changing the height of the layered lens

\begin{tabular}{|c|c|c|c|c|}
\hline$\alpha$ & $\mathbf{L}=\boldsymbol{\lambda}$ & $\mathbf{L}=\mathbf{1 . 5 5 \lambda}$ & $L=1.75 \lambda$ & $\mathbf{L}=\mathbf{2 \lambda}$ \\
\hline \multirow[t]{3}{*}{$\overline{0.11}$} & & & & \\
\hline & -1 & $-2+1=$ & 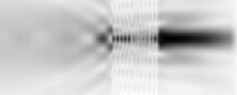 & $=$ \\
\hline & $\begin{array}{c}\text { DOF }=3.7 \lambda \\
F W H M=0.89 \lambda\end{array}$ & $\begin{array}{c}\text { DOF }=3.2 \lambda \\
F W H M=0.67 \lambda\end{array}$ & $\begin{array}{c}\text { DOF }=2.94 \lambda \\
F W H M=0.78 \lambda\end{array}$ & $\begin{array}{c}\text { DOF }=1.9 \lambda \\
F W H M=0.73 \lambda\end{array}$ \\
\hline \multirow[t]{3}{*}{0.12} & & & & \\
\hline & -1 & $-2-1=$ & zin:-ine & \\
\hline & $\begin{array}{c}\text { DOF }=3.73 \lambda \\
F W H M=0.85 \lambda\end{array}$ & $\begin{array}{c}\text { DOF }=2.85 \lambda \\
F W H M=0.68 \lambda\end{array}$ & $\begin{array}{c}\text { DOF }=2.64 \lambda \\
F W H M=0.87 \lambda\end{array}$ & $\begin{array}{c}\text { DOF }=0.93 \lambda \\
F W H M=0.79 \lambda\end{array}$ \\
\hline
\end{tabular}

When comparing rows of Table 1 it should be noted that the use of a layered lens provides a more narrow size of the focal spot, and when the value of $\alpha$ is higher than 0.12 , and more extended focal light segment. Let us consider in more detail the layered lens effect in changing its length along the axis of propagation of the laser beam for two cases mentioned earlier: $\alpha=0.11$ and $\alpha=0.12$ (Table 2). We consider the FWHM at the point of maximum intensity.

Table 2 shows that the increase in length of the lens leads to a reduction of the focal length for DOF. Increasing the lens length of an increase of the numerical aperture, only makes sense to a certain value (Figure 2). Table 2 shows that the increase in the 
length of more than $1.55 \lambda$ lens reduces the depth of focus at a constant value of FWHM.

Consider the change in the height of the diffraction axicon in case $\alpha=0.12$, i.e. at a numerical aperture of $\mathrm{NA}=0.64$. We varied the refractive index $n$. The height of the respective axicon considered on the basis of the phase shift at $\pi$ by the formula (8). Numerical simulation result is shown in Table 3.

Table 3. Diffraction of Gaussian beam on diffraction axicon with a change of the refractive index $n$

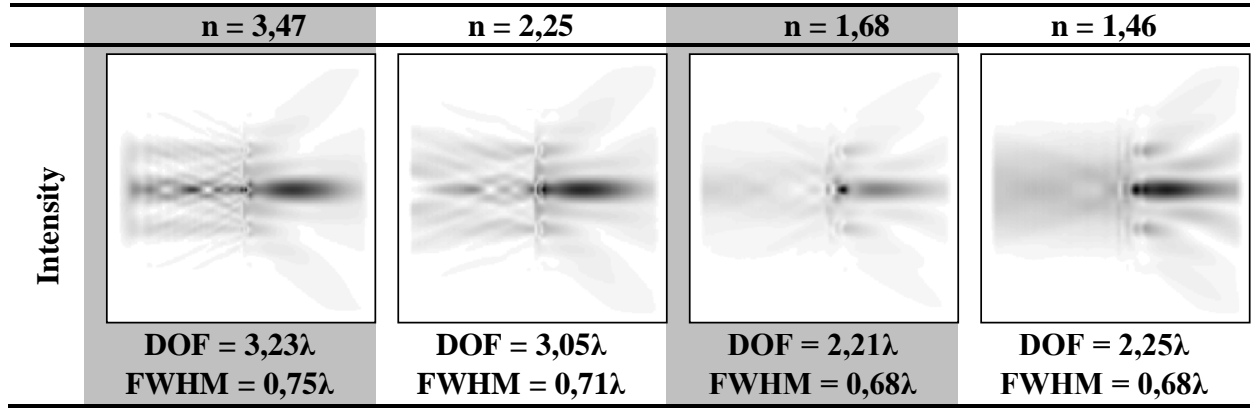

Decrease in the refractive index and simultaneously increase axicon relief leads to a reduction of the lengths of light segment. However, after a certain limiting value $(n=$ 1.68) DOF begins to increase again. It is also worth noting the reduction in the size of the focal spot with a decrease in the index of refraction of the axicon. However, it should be noted that after reaching a limiting value of the refractive index (in this case, when $n=1.68$ ) of the focal spot size is stabilized and becomes comparable to the previously discussed case of layered lenses.

\section{Conclusion}

Analytically and numerically using the finite-difference time-domain method we performed a comparative study of the diffraction of Gaussian beam by diffraction microaxicon and conical axicon, and gradient micro-lens consisting of sub-wavelength layers. The parameters under consideration the types of elements estimated on the depth of focus and a transverse dimension of beam.

Studies have shown that layered lens with linear variation of the refractive index has an advantage over diffraction axicon with the same numerical aperture, as it allows to form a narrower focal lengths. Increasing the numerical aperture of the axicon reduces the focal spot formed by them, but it is accompanied by a reduction of the light segment lengths. With a value of more than $\alpha=0.12$ (numerical aperture of more than 0.64) was obtained more extended light length segment for a layered lens.

By reducing the thickness of the layered lens is extended light segment and increases its width in the plane of maximum intensity along the propagation axis. After a certain point, in our case $1.55 \lambda$, there is the stabilization of transverse dimension with shortening the length of a segment. 
Studies on the reduction of the refractive index of the diffraction axicon show that after reaching a limiting value of the refractive index (in this case, when $n=1.68$ ) the focal spot size is stabilized and not decreases.

\section{Acknowledgment}

This work was supported by the Russian Foundation for Basic Research (grants 1607-00825a) and by the Ministry of Education and Science of Russian Federation.

\section{References}

1. Tomlinson WJ. Applications of GRIN-rod lenses in optical fiber communication systems. Applied Optics, 1980; 19(7): 1127-1138.

2. Tearney GJ, et al. Scanning single-mode fiber optic catheter-endoscope for optical coherence tomography. Optics Letters, 1996; 21: 543-545.

3. Kurt H, et al. The focusing effect of graded index photonic crystals. Applied Physics Letters, 2008; 93(17): 171108.

4. Kotlyar VV, Kovalev AA, Triandafilov YaR, Nalimov AG. Modes of planar gradientindex hyperbolic secant waveguide. Computer Optics, 2010; 34(2): 146-54. [in Russian]

5. Kotlyar VV, Kovalev AA, Nalimov AG. Gradient-index element of micro-optics for super-resolution. Computer Optics, 2009; 33(4): 369-78. [in Russian]

6. Emkey WL, Jack CA. Analysis and Evaluation of Graded-Index Fiber-Lenses. Journal of Lightwave Technology, 1987; LT-5: 1156-1164.

7. Chien HT, et al. The comparison between the graded photonic crystal coupler and various coupler. IEEE . Journal of Lightwave Technology, 2009; 27(14): 2570-2574.

8. Khonina SN, Striletz AS, Kovalev AA, Kotlyar VV. Propagation of laser vortex beams in a parabolic optical fiber. Proceedings of SPIE, 2010; 7523: 75230B-12p.

9. Khonina SN, Kazanskiy NL, and Soifer VA. Optical vortices in a fiber: mode division multiplexing and multimode self-imaging. Chapter 15 (26 p.) in "Recent progress in optical fiber research", ed. by M.Yasin, S.W. Harun, H. Arof, INTECH publisher, Croatia, 2012, $450 \mathrm{p}$.

10. Gilsdorf RW, Palais JC. Single-mode fiber coupling efficiency with graded-index rod lenses. Applied Optics, 1994; 33(16): 3440-3445.

11. Kotlyar MI, et al. Photonic crystal lens for coupling two waveguides. Applied Optics, 2009; 48(19): 3722-3730.

12. McLeod JH. The axicon: a new type of optical element. Journal of the Optical Society of America, 1954; 44(8): 592-597.

13. Durnin JE, Miceli JJ, Eberly JH. Diffraction-free beams. Physical Review Letters, 1987; 58: 1499-1501.

14. Kalosha VP, Golub I. Toward the subdiffraction focusing limit of optical superresolution. Optics Letters, 2007; 32(24): 3540-3542.

15. Khonina SN, Nesterenko DV, Morozov AA, Skidanov RV, Soifer VA. Narrowing of a light spot at diffraction of linearly-polarized beam on binary asymmetric axicons. Optical Memory and Neural Networks (Information Optics), 2012; 21(1):17-26.

16. Khonina SN, Savelyev DA. High-aperture binary axicons for the formation of the longitudinal electric field component on the optical axis for linear and circular polarizations of the 
illuminating beam. Journal of Experimental and Theoretical Physics, 2013; 117(4): 623630.

17. Ustinov AV, Khonina SN. Analysis of laser beam diffraction by axicon with the numerical aperture above limiting. Computer Optics, 2014; 38(2): 213-222.

18. Ustinov AV, Degtyarev SA, Khonina SN. Diffraction by a conical axicon considering multiple internal reflections. Computer Optics, 2015; 39(4): 500-507.

19. Lu JY, Greenleaf JF. Producing deep depth of field and depth-independent resolution in NDE with limited diffraction beams. Ultrasonic Imaging, 1993; 15(2): 134-149.

20. Turduev M, Giden IH, Kurt H. Design of flat lens-like graded index medium by photonic crystals: Exploring both low and high frequency regimes. Optics Communications, 2015; 339: 22-33.

21. Savelyev DA Simulation of linear gradient lenses for subwavelength focusing of Gaussian beams. CEUR Workshop Proceedings, 2015; 1490: 97-104. DOI: 10.18287/1613-00732015-1490-97-104

22. Savelyev DA, Ustinov AV, Khonina SN, Kazanskiy NL. Layered lens with a linear dependence of the refractive index change. Optical Technologies for Telecommunications 2015, Proc. SPIE, 2016; 9807: 98070P-1-7. DOI: 10.1117/12.2234404

23. Savelyev DA, Khonina SN. The calculation of the diffraction of the laser beams with a phase singularity on the micro-axicons with using high-performance computing. Journal of Physics: Conference Series, 2014; 490(1): 012213-1-5. 